\title{
Detection of Diabetic Retinopathy using Splat Feature Classification in Fundus Image
}

\author{
P. Latha, \\ PG Scholar \\ M.E Computer Science and Engineering, \\ Muthayammal Engineering College \\ Rasipuram- Namakkal
}

\author{
R. Vijayalakshmi, M.E., Ph.D., \\ Professor and Head, \\ Department of Computer Science and \\ Engineering, \\ Muthayammal Engineering College \\ Rasipuram-Namakkal
}

\begin{abstract}
Automated detection of retinal hemorrhages in fundus image $^{[2]}$ is crucial step towards early detection or screening is difficult among large population. A novel splat feature classification method is introduced to detect retinal hemorrhages. Classification is been achieved through supervised learning approaches. The performance of sensitivity and specificity is been improved while processing with retinal hemorrhages than with lesions. An area under receiver operating characteristics curve (ROC) of 0.96 can be achieved at splat level and 0.87 at image level.
\end{abstract}

\section{General Terms : Supervised Classification}

Keywords: Diabetic Retinopathy, Splat Feature Classification

\section{INTRODUCTION}

Diabetic retinopathy(DR) is a major public health issue since it can lead to blindness in patients with diabetes. Early treatment can prevent patients to become affected from this condition or atleast the progression of DR can be slowed down. The most common signs are microaneurysms, small hemorrhages, exudates, drusen and cottonwool spots. Because of the variability in appearance of these lesions, different techniques have been designed to detect each type separately. In most general DR Can be either proliferative or Nonproliferative DR.

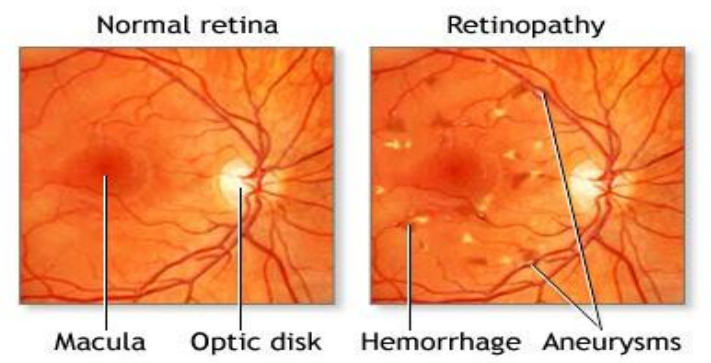

Fig 1. Normal and affected Retina

\section{EXISTING WORK}

In the existing work ${ }^{[1]}$, the process of detecting DR lesions is been accomplished by supervised classification which involves training classifiers using expert labeled target objects at pixel level. Features are extracted from each pixel and soft labels are assigned accordingly indicating the probability of pixel being one or part of the target object. Abnormal pixels are then combined into objects. There exists the limitations with this approach as follows

- It is expensive to acquire expert labeled reference standard for training and evaluation
- $\quad$ Specific training samples are intended to be both informative to the classification model and to diverse so that information provided by individual samples may overlap

- Large hemorrhages occur infrequently, have nonregular shape and can occur without accompanying other signs of DR. Because of low occurrence of lesions ${ }^{[3]}$, sensitivity for detection of large hemorrhages has negligible effect on unweighted performance metrics

Above problems can be resolved using higher level entity the splat and samples are to be upgraded for classification from pixel level to splat level, information is encoded at splat level with fewer disturbances from pixel level noise.

\section{PROPOSED WORK}

To present a supervised classification algorithm to detect retinal hemorrhages. Reference standards hemorrhage locations were delineated by a retinal specialist using splat based $^{[8]}$ image representation. Supervised classification predicts the likelihood of splats being hemorrhages with the optimal feature subset selected in a two step feature selection process from resulting hemorrhages map, a hemorrhage inde $\mathrm{x}^{[4]}$ is assigned as image level output.

\section{Splat Segmentation}

Splat based representation is an image re-sampling strategy onto an irregular grid. Background regions, with gradual variations in appearance tend to consists of fewer large splats while foreground regions consists of a large number of smaller splats. Splats are created by over segmenting images using watershed or toboggan algorithms. To create splats which preserve desired boundaries precisely, i.e., boundaries separating hemorrhages from retinal background, we perform a scale-specific image oversegmentation in two steps. We firstly aggregate gradient magnitudes of the contrast enhanced dark-bright opponency image at a range of scales for localization of contrast boundaries separating blood and retinal background. Next, the maximum of these gradients over scale-of-interest (SOI) is taken in performing watershed segmentation.

Assuming that we establish a scale-space representation of image I $(\mathrm{x}, \mathrm{y} ; \mathrm{s})$ with Gaussian kernels at SOI , the gradient magnitude $|\boldsymbol{\nabla} \boldsymbol{I}(\boldsymbol{x}, \boldsymbol{y}: \boldsymbol{S})|$ is computed from vertical and horizontal derivatives

$$
=\sqrt{I x(x, y ; s) 2+I y(x, y ; s) 2}
$$

The maximum of the gradient magnitude aggregated over the scale band $|\nabla I(x, y)|$ is

$$
\nabla I(x, y)|=\max | \nabla I(x, y ; s i)||
$$



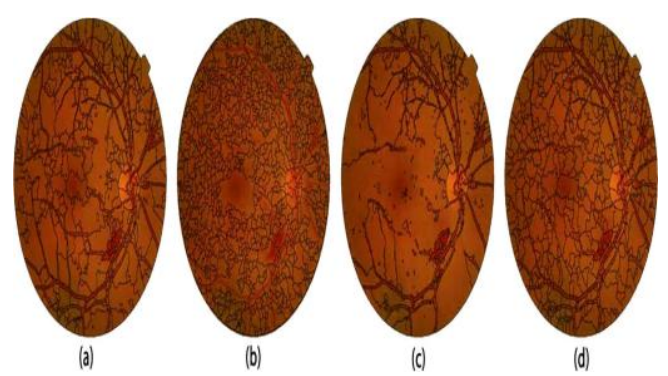

Fig 2.: watershed algorithm with topographic surface obtained as a) maximum of gradient magnitude from desired scale band b) pixel intensity c) gradient magnitude from a fine scale outside scale-of-interest d) gradient magnitude from a coarse scale outside scale-of-interest.

Supervised algorithms require labeled samples by experts, but it is expensive to acquire such data, because substantial time is required to delineate irregular boundaries of hemorrhages. To produce an image level reference standard, images with splat-based annotation from the expert i.e., images containing hemorrhages are given labels of "1" and the rest are given labels of " 0 " as they contain no hemorrhage splats.

\section{Edge Effect Removal}

As a preprocessing step, edge effects ${ }^{[5]}$ due to limited field of view (FOV) and vignetting in fundus photographs have to be addressed to suppress irrelevant responses during feature extraction. It is conventionally performed in two ways. One is to fill the region outside FOV with the mean color of the region within FOV. The other possibility is to mirror the FOV outside the FOV . To train a learning algorithm to detect hemorrhages, the mean color within splat $p$ is extracted in RGB color space and color opponency images for dark-bright, red-green and blue-yellow, comprising 6 color components $f_{p}^{c}$ in the splat feature space:

$$
f c p=\frac{1}{a p} \sum_{(x, y) \in p} I c(x, y)
$$

where $I c(x, y)$ is the image in one color plane and $\Omega p$ represents the set of pixels within splat $p$. Besides the absolute mean color, intensity variations across the fundus image have to be taken into consideration as well, because it can deteriorate the performance of color-based classification. Therefore differences between Gaussian smoothed images and the original one are computed at a number of scales $(\sigma=0.5$, $1,2,4,8,16$ ), which is expected to cover potential bandwidth of retinal hemorrhage boundaries. The mean difference along each splat boundary comprises additional features $f s p$ of that splat:

$$
f s p=\frac{1}{l p} \int[G s * I(x, y)-I(x, y)] d(x, y)
$$

where $\Omega p$ represents the boundary of splat $p$ with length $l p$. Feature $f_{s} p$ is also computed from all 6 color channels. Augmented with the mean color, the splat feature set has a total of 42 dimensions

\section{Splat Feature Extraction}

Given splats with their associated feature vectors and reference standard labels, a classifier can then be trained to detect target objects. In this study, two categories of features are extracted for splat-based hemorrhage detection as follows: 1) splat features aggregated from pixel-based responses; 2) splat wise features (no aggregation is required)

\section{Splat Feature Selection And Classification}

Two-Step Splat Feature Selection :Feature selection reduces the dimensionality of feature space by identifying relevant features and ignoring those irrelevant or redundant ones, which is particularly important to a higher separability between classes. There are two major approaches for feature selection: the filter approach and the wrapper approach ${ }^{[9]}$. The filter approach is fast, enabling their practical use on high dimensional feature spaces. It assesses individual feature separately without considering their interactions. The wrapper approach assesses different combinations of feature subsets tailored to a particular classification ${ }^{[7]}$ algorithm at the cost of longer computation time. To take advantage of both approaches, we use a two-step feature selection process a filter approach followed by a wrapper approach. The dataset is partitioned into a training set and a testing set. Feature selection evaluates discrimination power of candidate features according to reference standard labels of training set and comes up with an optimal subset as the input to a classifier. The accuracy of splat labels predicted by kNN classifier is assessed using leave-one-out cross-validation.

To eliminate spurious responses in hemorrhageness map $h$, firstly low probability responses are suppressed

$$
\begin{gathered}
\boldsymbol{h}(\boldsymbol{x}, \boldsymbol{y})=\boldsymbol{h}(\boldsymbol{x}, \boldsymbol{y}) \text { if } \boldsymbol{h}(\boldsymbol{x}, \boldsymbol{y}) \geq \boldsymbol{h} 0 \\
\text { else } 0
\end{gathered}
$$

where $\mathrm{h} 0$ is the predefined threshold. Secondly, given a limited number of hemorrhage splats coming out from the first step, those neighboring ones are merged together to form objects. Objects with small areas are removed because they are more likely to be red lesions or microaneurysms, which are supposed to be detected by separate detectors. Thirdly, splats formed by the fovea, whose locations are detected automatically, are masked out to suppress potential false positives.

\section{Image Level Hemorrhage Index}

The goal of the splat feature classification is to develop a large retinal hemorrhage detector, indicating whether or not an image is normal, i.e. free of hemorrhages, or abnormal, i.e. containing one or more hemorrhages. When the posterior probability of each splat being blood regions is determined, a hemorrhage-likeness map pblood can be created for each testing image. To upgrade this hemorrhage-likeness map to image level decision of the existence of retinal hemorrhages, pblood has to be turned into a single index which indicates the likelihood that the corresponding fundus image contains hemorrhages.

To extract possible candidate hemorrhage locations, pblood was first thresholded to produce a binary image. In order to determine an appropriate threshold, the mean hemorrhagelikeness of blood vessel splats labeled by the vessel probability map pvessel was examined. This gave an indication of the mean probability value of blood splats that could be detected at a certain threshold. Further experimentation showed that 0.88 gave a good separability of blood splats and the rest of the retina. After thresholding, a binary image containing hemorrhages (if any) and the vascular tree was obtained. By removing vessel splats, the remaining responses indicate the presence of hemorrhages. Neighboring hemorrhage splats are merged together to form one object. The hemorrhage index is computed as the mean 
probability of the hemorrhage splats, weighted by the area of the largest hemorrhage among them.

\section{EXPERIMENTS AND RESULTS}

Data Collection :A set of 1200 fundus photographs from the publicly available Messidor database was used, which were acquired by three ophthalmologic departments using color video 3CCD camera

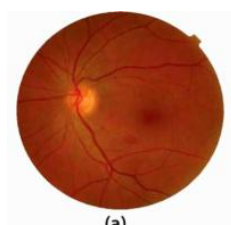

(a)

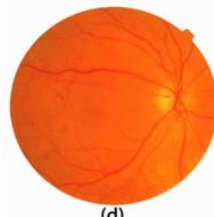

(d)

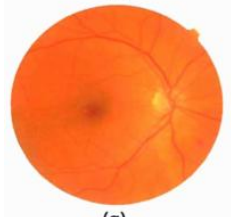

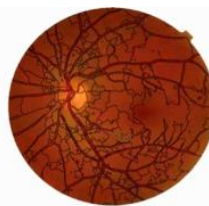

(b)

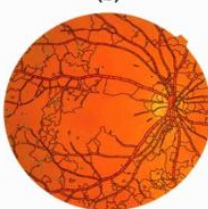

(e)

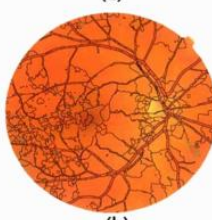

(h)
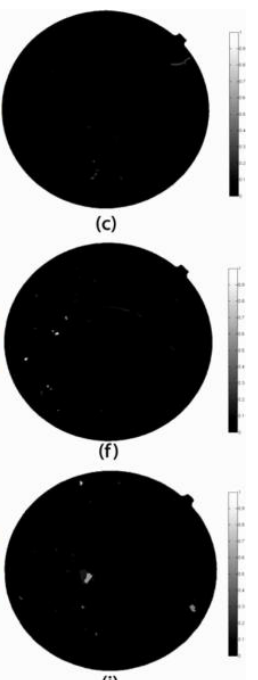

Fig 3: Fundus Image and their hemorrhages maps selected from splat-based feature classification. a) Fundus image 1 b) expert annotation $1 \mathrm{c}$ ) hemorrhageness map 1 d)Fundus image 2 e) expert annotation 2 f) hemorrhageness map 2 g) Fundus image 3 h) expert annotation 3 i) hemorrhageness map 3

The images were captured using 8 bits per color plane at $1440960,2240 \quad 1488$ or 23041536 pixels. In a preprocessing step, the FOV was detected automatically and the images were rescaled to $1026 \quad 681$ pixels with the FOV approximately 630 pixels in diameter across the entire dataset. All personally identifiable information was stripped so that only the raw image data was available. A subset of the first 300 images in the Messidor dataset, not used in the development or testing of any other part of the system, provided the training splats. The testing set contains splats created from the rest of 900 images which is entirely in- dependent of the training set. As the number of large hemorrhages is far less than what required to train the classifier, the expert also annotated images which contain small or medium hemorrhages. Overall, 138 of 1200 images were annotated by expert (27 from the training set and 111 from the testing set).

Analysis of Misclassification :Patient safety is the most important factor in designing and improving a system which can be translated into practice. Thus, top false negative (FN) cases, i.e., cases which do contain hemorrhages but assigned very low hemorrhage indices, were reviewed. FNs include elongate hemorrhages present in the neighborhood or even connected with the vasculature. We also reviewed top false positive (FP) cases, i.e., cases which actually contain no hemorrhages but assigned high hemorrhage indices Major causes of misclassification (presence/absence) or discrepancy of the severity of hemorrhages (total area) include the presence of clearly visible choroid vessels, retinal background surrounded by a cluster of bright lesions, significantly different appearances between arteries and veins, or white reflections visible in young fundus.

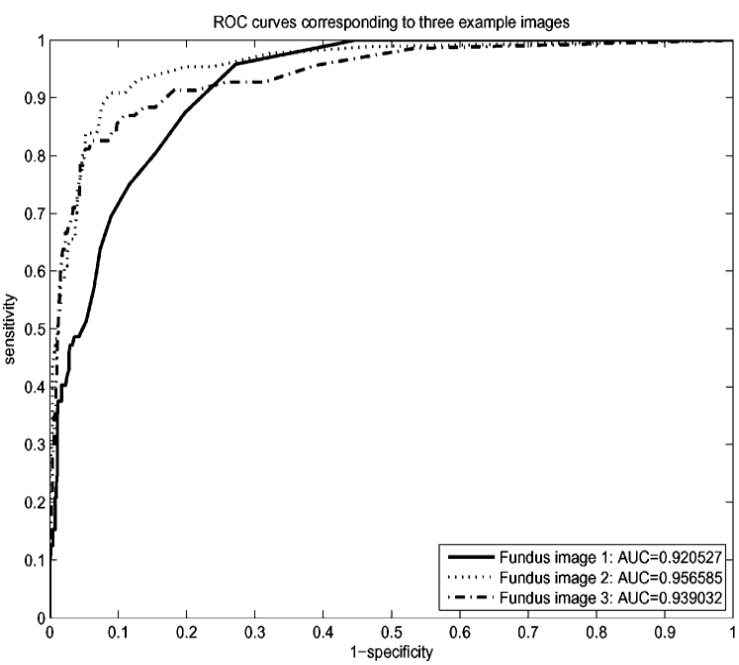

(a)

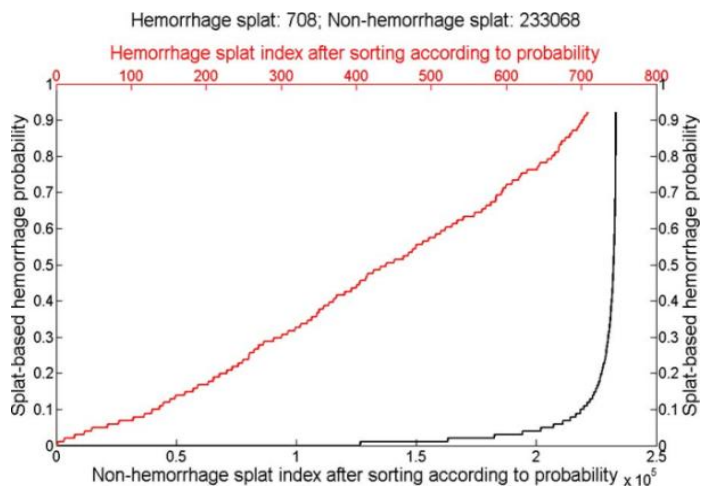

(b)

Fig 4.a) Splat Based ROC curves corresponding to images b)Probabilities assigned to hemorrhage splats and nonhemorrhage splats

\section{CONCLUSION}

We present a splat-based feature classification algorithm with application to large, irregular hemorrhage detection in fundus photographs. Neighboring pixels with similar intensity are grouped into nonoverlapping splats. A set of features is extracted from each splat to describe its characteristics. These splats are taken as samples for supervised classification in a selected feature space. The algorithm is validated on the publicly available Messidor dataset with an area under the ROC curve (AUC) of 0.96 at the splat level. At the image level, an AUC of 0.87 was achieved. Large, irregular hemorrhage detection is a challenging problem due to the fact that they are rare and irregular in shape with substantial variability in appearance. To assess performances of an automated system, we conducted image level ROC and FROC analysis, showing that the system can operate at a sensitivity of $93 \%$ and specificity of $66 \%$, if the threshold is set to produce an average of 0.2 FPs on a per image basis.

\section{ACKNOWLEDGMENT}

The authors would like to thank the Messidor program partners for kindly providing their database (see http://messidor. crihan.fr). 


\section{REFERENCES}

[1] Li Tang, Meindert Niemeijer, Joseph M. Reinhardt, Mona K. Garvin, and Michael D. Abràmoff*, "Splat Feature Classification With Application to Retinal Hemorrhage Detection in Fundus Images" IEEE Trans on Med img. Vol 32,pp.364-375 Feb 2011.

[2] M. D. Abràmoff, J. M. Reinhardt, S. R. Russell, J. C. Folk, V. B. Mahajan, M. Niemeijer, and G. Quellec, "Automated early detection of diabetic retinopathy" Ophthalmology, no. 6, pp. 1147-1154, Apr.

[3] M. Niemeijer, B. van Ginneken, J. Staal, M. S. A. Suttorp-Schulten, and M. D. Abràmoff, "Automatic detection of red lesions in digital color fundus photographs," IEEE Trans. Med. Imag., vol. 24, no. 5, pp. 584-592, May 2005.

[4] P. Jitpakdee, P. Aimmanee, and B. Uyyanonvara, "A survey on hemorrhage detection in diabetic retinopathy retinal images," in Proc. 9th Int. Conf. Elect. Eng./Electron., Comput., Telecommun. Inf. Technol. (ECTI-CON), Bangkok, Thailand, 2012, pp. 1-4, vol..
[5] Vijayalakshmi R and Selvarajan S, (2014) "Comparative Studies on Canny and Sobel Edge Detection Techniques for Diabetic Retinopathy Eye Image" International Journal of Soft Computing, Vol. 9, Issue. 4, pp. 213-218.

[6] M. Abràmoff, M. Garvin, and M. Sonka, "Retinal imaging and image analysis," IEEE Rev. Biomed. Eng., vol. 3, pp. 169-208, 2010.

[7] X. Ren and J. Malik, "Learning a classification model for segmentation," in Int. Conf. Comput. Vis., 2003, vol. 1, pp. 10-17.

[8] L. Tang, M. Niemeijer, and M. Abràmoff, "Splat feature classification: Detection of the presence of large retinal hemorrhages," in Proc. IEEE 8th Int. Symp. Biomed. Imag. (ISBI), 2011, pp. 681-684.

[9] R. Kohavi and G. John, "Wrappers for feature subset selection," Artif.Intell., vol. 97, no. 1-2, pp. 272-324, 1997. 